\title{
'Getting to Know Me': The second phase roll-out of a training programme for supporting people with dementia in general hospitals
}

Ruth Elvish, Simon Burrow, Rosanne Cawley, Kathryn Harney, Julie Gregory, Mark Pilling and John Keady

Corresponding author: Ruth Elvish

School of Nursing, Midwifery and Social Work, University of Manchester, Manchester M13 9PL UK Tel: 01670676080 Email: ruth.elvish@googlemail.com

Simon Burrow

School of Nursing, Midwifery and Social Work, University of Manchester, Manchester M13 9PL UK Tel: 01613067835 Email:

simon.burrow@manchester.ac.uk

Rosanne Cawley

Salford Learning Disability Team, Greater Manchester West Mental Health NHS Foundation Trust, Salford Civic Centre $2^{\text {nd }}$ Floor, Chorley Road, Swinton M27 5FJ UK Tel: 01617032124 Email: rosanne.cawley@salford.gov.uk

Kathryn Harney

Research and Development Office, Greater Manchester West Mental Health NHS Foundation Trust, Trust Headquarters, Bury New Road, Prestwich, Manchester M25 3BL UK Tel: 01617723591 kathryn.harney@gmw.nhs.uk

Julie Gregory

School of Nursing, Midwifery and Social Work, University of Manchester, Manchester M13 9PL UK Tel: 01613067825 Email: julie.gregory@manchester.ac.uk

Mark Pilling

School of Nursing, Midwifery and Social Work, University of Manchester, Manchester M13 9PL UK Tel: 01613067778 Email: mark.pilling@manchester.ac.uk

John Keady

School of Nursing, Midwifery and Social Work, University of Manchester, Manchester M13 9PL UK Tel: 01613067854 Email: john.keady@manchester.ac.uk 


\section{Acknowledgements}

Rilwan Adebiyi, Gwen Ainsworth, Danielle Beswick, Brian Briggs, Kati Edwards, Emily Feilding, Pat Graham, Mike Howorth, Ann Johnson, Harry Johnson, Nicola Johnson, Stephanie Jolly, Jenna King, Janice McGrory, Andrew Powell, Abi Tarran-Jones, Pamela Roach, Rebecca Wild, Gillian Zajac-Roles

Word Count: 


\begin{abstract}
Objective: The aims of this study were to evaluate a second phase roll-out of a dementia care training programme for general hospital staff: The 'Getting to Know Me' programme. The study also aimed to further develop two outcome scales: the CODE scale for measuring confidence in working with people with dementia and the KIDE scale for measuring knowledge in dementia.
\end{abstract}

Method: Following a 'training the trainers' phase, the study involved the delivery of the "Getting to Know Me" training programme to a large number of staff $(n=517)$ across three NHS Trusts situated in North-West England. The impact of the programme was evaluated using a pre-post design which explored: i) changes in confidence in dementia; ii) changes in knowledge in dementia; and iii) changes in beliefs about behavious that challenge.

Results: Statistically significant change was identified between pre-post training on all outcome measures (CODE: 7 point increase, $p<0.001$; KIDE: 2 point increase $p<0.001$; controllability beliefs scale: 4 point decrease, $p<0.001)$. Medium to large effect sizes were demonstrated on all outcome measures, demonstrating clinically meaningful change on all outcomes. The psychometric properties of the CODE and KIDE scales are reported.

Discussion: The "Getting to Know Me" training programme was undertaken with a large number of general hospital staff from a range of disciplines. Staff knowledge in dementia and confidence in working with people with dementia significantly increased following attendance at the training sessions. The findings are consistent with preliminary findings (Elvish et al., 2014) and strengthen current knowledge about the impact of dementia care training in general hospitals. The CODE and KIDE scales continue to demonstrate psychometrically sound properties and demonstrate utility in the field of dementia research. 
Key words: acute care; dementia; general hospital; scale development; staff training Introduction

The number of people living with dementia across the globe is estimated at 35.6 million (Prince et al., 2013); this number is expected to double in the next twenty years (Knapp, Prince, \& Albanese, 2007). As older adults are admitted to hospital more frequently and for longer periods than the younger population (Trueland, 2014), it is not surprising to find that up to $25 \%$ of general hospital beds are occupied by people with dementia and that more than $97 \%$ of general hospital staff report having cared for patients with dementia (Alzehimer's Society, 2009). Despite these figures, in 2011 it was highlighted that there was no mandatory dementia training in 95\% of general hospitals (Royal College of Psychiatrists, 2011) and a growing body of evidence suggests that dementia care training should be improved for all general hospital staff (Department of Health, 2009; Tullo \& Allan, 2011). The picture in the UK is beginning to shift as dementia training is rolled out across the NHS to help staff to identify signs of dementia and to improve communication skills (Department of Health, 2014). Providing an excellent standard of care for people with dementia whilst they are patients within general hospitals is now a current priority within health and social care arenas (Department of Health, 2015).

Despite the fact that the majority of current literature acknowledges the importance of person-centred care for people with dementia when they are in general hospital (Dewing \& Dijk, 2014), a report by the Alzheimer's Society (2009) highlights that there are various barriers which pose a challenge to implementing this. These barriers include: insufficient training and education (Moyle, Borbasi, Wallis, Olorenshaw, \& Gracia, 2010), lack of competence and confidence in managing behaviour that challenges (Atkin, Holmes, \& Martin, 2005), and attributing 'fault' to a person with dementia for behaviour that challenges (Smythe et al., 2014). A further barrier is the belief of many general hospital staff that their role is to focus on physical care rather than on needs arising because of a person's cognitive impairment (Calnan, Tadd, Calnan, Hillman, Read, \& Bayer, 2012). Charter and 
Hughes (2012) argue that this lack of priority placed on the specific needs of people with dementia is, at least partly, due to lack of specialised training and education.

A recent review by Dewing and Dijk (2014) highlights the lack of agreement about what would lead to improvements in the care of people with dementia in general hospitals. Some studies argue that an improved staff to patient ratio would improve care (Gladman et al., 2012), whereas others argue that higher staffing levels do not necessarily translate into meaningful interactions (Edvardsson, Sandman, \& Rasmussen, 2011). However, there does seem to be agreement that when a person with dementia is in hospital they want staff to understand their needs and routines (Keenan et al., 2011), connect with them (Bridges, Flatley, Meyer \& Nicholson, 2009), and give them a feeling of control over their situation (Gladman et al., 2012).

There have been a number of strategies used within general hospital settings to address the issues outlined above. Dementia training programmes with general hospital staff have led to a number of outcomes, including improvements in staff knowledge and confidence, and shifts towards more person-centred perspectives about behaviour that challenges (Elvish et al., 2014; Galvin et al., 2010). Dewing \& Dijk (2014) discuss other strategies as mental health liaison teams and specialist units for the care of people with dementia requiring acute care (Nichols \& Heller, 2002). However, as Dewing \& Dijk point out, mental health liaison teams for older people in general hospital often provide crisis intervention rather than training for staff caring for people with dementia in general hospital on a daily basis (Holmes et al., 2010). Building on work to date, there is clearly much to be done within both clinical and research settings to address the issue of caring for people with dementia within a general hospital environment.

\section{Aims of the current project}

Our initial study (Elvish et al., 2014) evaluated a training programme for general hospital staff: the 'Getting to Know Me' programme. The evaluation was conducted within one NHS site and suggested that confidence in working with people with dementia and knowledge in dementia increased in staff members $(n=72)$ following 
completion of the programme (Elvish et al., 2014). In addition, following the training, beliefs about challenging behaviour shifted towards a more person-centred perspective on behaviours that challenge, although these shifts were relatively small. There were a number of limitations to the initial study. These were: i) a significant amount of the training was delivered by the authors of the training materials; ii) the implementation of the training programme was limited to one hospital site; and iii) the study used new questionnaires which did not have established psychometric properties.

The current project aimed to address the limitations identified above: i) by involving a 'training the trainers' phase in which staff from participating NHS Trusts were trained by staff from the University of Manchester project team in the use of the "Getting to Know Me" training programme. The programme was then delivered by these NHS staff rather than the author(s) of the training materials; ii) by recruiting across three NHS Trusts; and iii) by utilising the questionnaires from the initial project and undertaking analysis to further explore their psychometric properties.

\section{Methods}

Three NHS Trusts within North-West England agreed to participate in the current project. The study was given a favourable opinion by the University of Manchester ethics committee, and approval to undertake the project was given by the Research and Development Offices from: (i) Salford Royal NHS Foundation Trust; (ii) Central Manchester University Hospitals NHS Foundation Trust; and (iii) Trafford Healthcare NHS Foundation Trust.

\section{Overview}

The design of the current study was broadly consistent with the design of the initial evaluation of the "Getting to Know Me" training programme (Elvish et al. 2014). A pre-post design assessed changes following completion of the "Getting to Know Me" training programme in: i) confidence in working with people with dementia; ii) knowledge in dementia; and iii) beliefs about behaviour that challenges. Outcome measures were administered at two time points: immediately before the training 
(time point one), and immediately after the training had been completed (time point two). Two of the outcome measures used (the Confidence in Dementia (CODE) scale and the Knowledge in Dementia (KIDE) scale) were piloted in the initial study and showed promising psychometric properties. The current evaluation utilised classical test construction methods to undertake further psychometric analysis of these measures.

\section{Participants}

Participants were required to be staff members working in one of three NHS Trusts that were participating in the project. As identified by senior NHS staff linked to the study, departments deemed to provide care to patient groups that included people with dementia were identified and staff were drawn from the following services: Stroke Rehabilitation, Cardiac Rehabilitation, Day Services, General Out-Patients, Orthopaedics, Heart care Unit, Neurology Unit, Dermatology, Rheumatology, Acute Respiratory, Acute Elderly, Endoscopy, Discharge Assessment Team, Accident \& Emergency, Maternity, Complex Care, Medical Assessment, Observational Medical Unit, Safeguarding, Neurosurgery, Gastro-Surgery, and Surgical High Dependency.

\section{Scale development and outcome measures}

\section{Confidence in Dementia (CODE) Scale}

This nine-item self-report questionnaire was used to measure confidence in working with people with dementia. The questionnaire is scored on a five point Likert scale with anchored ratings of 'not able', 'somewhat able', and 'very able'. This meant that it was possible to gain a total score between 9 and 45, with a higher score representing better confidence in working with people with dementia.

Psychometric analysis was undertaken on the CODE scale by combining data from the initial study (Elvish et al., 2014) with data from the current study. Analysis was therefore undertaken on data from 696 participants. The Cronbach alpha value for the scale was 0.88 and the overall KMO was 0.89 , suggesting that the scale had high internal consistency without too much item redundancy and excellent sample 
adequacy. These values are very similar to the previous study (alpha=0.91, KMO=0.90, n=72) (Nunally, 1978; Streiner and Norman, 2003).

\section{Knowledge in Dementia (KIDE) Scale}

This 16 -item self-report questionnaire was used to measure knowledge in dementia. The questionnaire is scored on an agree/disagree scale; 'agree' responses are given a score of 1 , 'disagree' responses are given a score of 0 . This meant that it was possible to gain a total score between 0 and 16 , a higher score being representative of better knowledge about dementia.

Psychometric analysis was undertaken on the KIDE scale by combining data from the initial study (Elvish et al., 2014) with data from the current study. The Cronbach alpha value of the scale was 0.66 and the overall $\mathrm{KMO}$ was 0.76 , again suggesting that the scale had good internal consistency without too much item redundancy and excellent sample adequacy. Again, these values are very similar to the previous study (alpha=0.72, KMO=0.70) (Nunally, 1978; Streiner \& Norman, 2003).

\section{Controllability Beliefs Scale}

This 15 -item self-report questionnaire was used to measure staff beliefs about the controllability of behaviour that challenges. The scale is based on the theory that attributions of controllability determine staff responses. The questionnaire is measured on a five point scale with anchored ratings of 'agree strongly', 'agree slightly', 'unsure', 'disagree slightly', and 'disagree strongly'. This meant that it was possible to gain a total score of between 15 and 75, a higher score being representative of a more person-centred approach to understanding behaviour that challenges. Cronbach's alpha for the scale is 0.8 , suggesting a high degree of internal consistency (Dagnan, Grant \& McDonnell, 2004).

\section{Statistical Procedures}

A Kruskal Wallis test was completed on the data at both time points in order to ascertain if there were significant differences between Trust sites \& to determine if the Trusts should be pooled. 
For analysis of pre-post change on total scores of the CODE, KIDE and Controllability Beliefs scales, a Wilcoxon signed-rank test was used to compare paired data between time-points because the change score was not Normally distributed. Each outcome measure was treated as independent of the others. The alpha level was set at 0.025 (two-sided test) for all analyses, and $p$ values of less than or equal to 0.025 were considered statistically significant. These values were chosen because an interim analysis had been undertaken at $n=413$ prior to the final analysis. This analysis was undertaken to provide data for a research grant proposal.

An effect size of above 0.30 was a priori considered clinically significant, and effect sizes of $0.10-0.30$ were considered as approaching clinical significance. These cutpoints are consistent with effect sizes reported for staff training interventions. Effect sizes for Wilcoxon signed-rank tests were calculated using the formula $r=z / V n$ (Field, 2005).

\section{Sample Size and Power}

Power was calculated based on published psychometrics of the primary outcome measure, the controllability beliefs scale (Dagnan, Grant, \& McDonnell, 2004). This study reports a mean of 49.47 and a standard deviation of 12.96. Assuming a medium level of correlation $r=0.3$ (Cohen, 1992) between values of the primary outcome before and after the intervention, and the same standard deviations before and after, using a 2-sided paired t-test at significance level 0.05 and $80 \%$ power, the calculated sample size needed to detect a before-after difference of five was 76 participants.

As the study was undertaken with staff from three NHS Trusts, this was a cluster trial. Assuming an intra-class correlation (rho) of 0.008, then the design effect is 1.6. We therefore required an average of 122 participants to be recruited per Trust. 
In order to allow for an attrition rate of $45 \%$ (based on the initial study where $72 / 125=58 \%$ completed the study, Elvish et al., 2014), the recruitment aim for the study was 222 participants per Trust, or 666 participants in total.

\section{The Intervention}

Figure 1 provides an overview of the six-hour training programme and its materials (all of which are available for free download from www.gmhiec.org.uk). For further details about the background and development of the 'Getting to Know Me' training programme, please see Elvish et al. (2014).

The training was designed to be delivered flexibly; in the current study it was delivered in one full training day. Trainers were members of staff from each of the three Trusts involved in the current project. Trainers received a two-day training course in the use of the "Getting to Know Me" programme. This training was undertaken by two authors (SB \& JG) from the University of Manchester. Thirty-five trainers were trained (nurses $=20$, mental health practitioners $=5$, clinical educators or practice trainers $=6$, and unknown $=4$ ).

INSERT FIGURE 1 ABOUT HERE

\section{Results}

\section{Participants}

Figure 2 is a diagrammatic representation of the flow of participants through the study.

\section{INSERT FIGURE 2 ABOUT HERE}

The total number of staff that completed the training programme was 607 . Five hundred and seventeen participants consented to take part in the project, however, participants with missing data were excluded from the analysis. Thus, CODES data from 480 participants was deemed eligible and included for purposes of Pre-Post 
analysis. Participants in the study analysis therefore represented $79 \%$ of the total sample trained. This attrition rate (21\%) was lower than the predicted drop-out rate, which was based on the attrition rate from the original study (Elvish et al., 2014). It is likely that the attrition rate was lower in the present study because the training programme was undertaken in one day rather than in a number of sessions across time.

Of the study sample, $52 \%(n=242)$ were nurses, $22 \%(n=103)$ were healthcare assistants, $4 \%(n=18)$ were physiotherapists/assistant therapists/occupational therapists, $4 \%(n=17)$ were cadet nurses, $2 \%(n=10)$ were practitioners and assistant practitioners, $1 \%(n=6)$ were student nurses, and $1 \%(n=6)$ were housekeeping staff. A further $5 \%(n=22)$ comprised a pharmacist, clinical educators, dieticians, a podiatrist and a dentist, and $9 \%(n=44)$ were unknown. Eighty-two percent of participants were female, and the median length of time employed within health care services was eight years. Sixty-eight percent of the sample said they had received no training in dementia care. Twenty percent said they had received some training; of these, $12 \%$ stated they had a basic knowledge, $7 \%$ stated they had a good amount of knowledge, and 1\% reported a high level of specialist training (eg. a degree level course).

\section{Scale development}

The CODE scale and the KIDE scale are presented in tables one and two respectively. Readers are directed to Elvish et al. (2014) for detailed descriptions of the questionnaires and initial psychometric findings.

Current psychometric analysis was undertaken on a total of $n=696$ through combining data from the current study and the initial study (Figure 3).

\section{INSERT FIGURE 3 ABOUT HERE}

The Cronbach alpha value for the CODE scale was 0.88 and the overall KMO was 0.89. The Cronbach alpha for the KIDE scale was 0.66 and the overall KMO was 0.76 . 
These results suggest that both scales have good internal consistency without too much item redundancy (Nunally, 1978; Streiner \& Norman, 2003; Pett, Lackley, \& Sullivan, 2003).

Based on initial responses to the CODE scale, our current interpretation of the cutoff points within the scale are as follows: $0-18=$ not confident, $19-35=$ somewhat confident, $36-45=$ very confident. Table 3 depicts the numbers of staff members that fell within each of these categories pre and post-training in the current study.

INSERT TABLE 1, 2 AND 3 ABOUT HERE

\section{Pre-post analysis}

A Kruskal Wallis test was performed on the data at both time points in order to ascertain if there were significant differences between Trust sites. For both the KIDE $(p=0.81)$ and CODE $(p=0.87)$ scale there was no evidence that the change scores differed between Trusts. Therefore it was appropriate that the data from all three Trust sites was combined in the analysis.

All results were consistent with those found in the interim analysis.

Pre-post analysis on total CODE scores $(n=480)$ revealed that confidence levels were significantly higher immediately after the training (Median $=36$ ) than immediately before the training (Median $=29), z=-14.68 p<0.001$ (Wilcoxon), with effect size $r=-$ 0.96. The median difference was seven, and the $95 \%$ bootstrap confidence interval (CI) for the median was $[6,7]$ using the BCa method (adjusted bootstrap percentile). This suggested that staff confidence in working with people with dementia had increased following the training sessions, and interpretation consistent with other research indicates this is a meaningful size of change (Galvin et al., 2010).

Pre-post analysis on total KIDE scores $(n=476)$ revealed that levels of knowledge were significantly higher immediately after the training (Median $=14.0$ ) than 
immediately before the training (Median $=12.0$ ), $z=-13.59 p<0.001$ (Wilcoxon), with effect size $r=-0.8$. The median difference was small at two, and the $95 \%$ bootstrap $\mathrm{Cl}$ for the median was $[1,2]$. This suggested that staff knowledge of dementia had improved following the training sessions, and in line with research within this area this is interpreted as a meaningful size of change (Galvin et al., 2010; Rapp et al., 1998).

A comparison of total scores on the controllability beliefs scale $(n=471)$ immediately before the training (Median=27) and immediately after the training (Median=21) revealed that there was a significant decrease in scores in the post-training condition $z=-11.06 p<0.001$ (Wilcoxon), with effect size $r=0.51$. The median difference was minus four, and the $95 \%$ bootstrap $\mathrm{Cl}$ for the median was $[-4,-5]$. This result pointed to staff holding a more person-centred perspective on challenging behaviour following the training, and in line with research within this area this is interpreted as a meaningful size of change (Galvin et al., 2010; Rapp et al., 1998).

\section{Discussion}

This paper has presented the evaluation of a second phase roll-out of a dementia care training programme for general hospital staff; the "Getting to Know Me" training programme. In addition to exploring the impact of the programme on a range of staff outcomes, the study also comprised the further development of two outcome measures: the CODE and KIDE scales.

After undertaking the training programme, knowledge in dementia and confidence in working with people with dementia increased. Beliefs about challenging behaviour also moved towards a more person-centred perspective on behaviours that challenge.

Over half of the staff trained were from a nursing background. Whilst the proportion of nurses trained is likely to reflect the high proportion of the general hospital workforce they comprise, it is also interesting to note that the majority of trainers 
were also nurses. Should future hospitals wish to implement the "Getting to Know $\mathrm{Me}$ " programme with a range of staff, a point of reflection may be the impact of the professional affiliation of the trainers on the staff who are encouraged and motivated to engage with the training.

The CODE and KIDE scales both have good internal consistency. Changes in scores on the scales suggested that staff knowledge and confidence had improved. This was in line with the hypotheses of the study, indicating that change measured was in the predicted direction. This data supports findings from the initial study (Elvish et al., 2014) that both questionnaires have good criterion validity.

Before the training programme, as measured by the CODE scale, the majority of staff described their confidence in working with people with dementia as 'somewhat confident'. Following the training, the majority of staff described themselves as 'very confident' in working in this area. This is encouraging, and suggests that the training resulted in meaningful changes in confidence.

As this paper reports on a second roll-out of the "Getting to Know Me" training programme, it is useful to draw overall reflections from both the initial study (Elvish et al., 2014) and the current study. Of note: i) the size of the change score on the CODE scale (difference in medians of 7) was similar to that observed in the previous evaluation (difference in medians of 6). This suggests that the impact of the second phase roll-out was consistent with the original evaluation, despite being delivered by a range of trainers. This also suggests that the CODE scale is a reliable measure; ii) the size of the change score on the controllability beliefs scale was larger in the current study than in the initial evaluation, making the change in the present study consistent with interpretations of clinically meaningful change. We are therefore able to draw stronger conclusions from the present study regarding the impact of our training on attitudes towards behaviour that challenges; iii) the current roll-out delivered the training in one full day, whereas the initial roll-out was predominantly delivered in four parts. The impact of this is unclear, however, further exploration of this in future studies would be helpful; iv) the initial study trained staff working on 
wards that predominantly provided care for older people. The present study addressed a much wider staff group yet yielded consistent results; and v) in line with studies which highlight the importance of the views of people with dementia to inform education (Charter \& Hughes, 2012), significant contributions were made to the development of the training programme by people with dementia and relatives. Service-user input in the field of dementia care remains an issue that is not always addressed well, therefore these contributions to the project are of extreme value and importance.

\section{Limitations}

The present study did not employ a control group and therefore limited conclusions can be drawn about the cause of change. Further studies to explore the impact of dementia care training within general hospital staff should consider the use of a control group.

It remains a challenge to find the most effective and efficient way of delivering training to staff who work within busy hospital environments. Future studies would benefit from identifying the elements of training programmes that are most effective in creating change so that programmes can be further developed in line with this. Studies using e-learning $v$ face to face training may be of particular benefit within the current climate.

Finally, the current study does not assess the impact of training on direct patient care and patient outcomes. Our work suggests that future studies should explore how changes in staff confidence, knowledge and attitudes impact on direct patient care.

\section{Conclusion}

This second phase roll-out of the 'Getting to Know Me' programme has provided evidence to strengthen current knowledge about the impact of dementia care training in general hospitals. Despite a number of (deliberate) differences to the 
original study in the ways in which the training was delivered, the current findings are consistent with initial findings that staff are more confident and have better knowledge about dementia following completion of the 'Getting to Know Me' training programme. As highlighted in a number of studies to date, we are reaching a point where we are able to further explore the impact of training on direct patient care and patient outcomes. We are in a time where the emphasis on dementia care across society means that the care of people with dementia in the general hospital setting spans a number of academic and clinical disciplines; a variety of research studies and clinical interventions should therefore continue to reveal the impact of current changes in the way that dementia care is addressed within the general hospital.

Table 1. Items of the Confidence in Dementia (CODE) Scale

\begin{tabular}{|c|c|}
\hline 1 & I feel able to identify when a person may have a dementia \\
\hline 2 & $\begin{array}{l}\text { I feel able to understand the needs of a person with dementia when they } \\
\text { can communicate well verbally }\end{array}$ \\
\hline 3 & $\begin{array}{l}\text { I feel able to understand the needs of a person with dementia when they } \\
\text { cannot communicate well verbally }\end{array}$ \\
\hline 4 & $\begin{array}{l}\text { I feel able to interact with a person with dementia when they can } \\
\text { communicate well verbally }\end{array}$ \\
\hline
\end{tabular}




\begin{tabular}{|l|l|}
\hline $\mathbf{5}$ & $\begin{array}{l}\text { I feel able to interact with a person with dementia when they cannot } \\
\text { communicate well verbally }\end{array}$ \\
\hline $\mathbf{6}$ & $\begin{array}{l}\text { I feel able to manage situations when a person with dementia becomes } \\
\text { agitated }\end{array}$ \\
\hline $\mathbf{7}$ & $\begin{array}{l}\text { I feel able to gather relevant information to understand the needs of a } \\
\text { person with dementia }\end{array}$ \\
\hline $\mathbf{8}$ & $\begin{array}{l}\text { I feel able to help a person with dementia feel safe during their stay in } \\
\text { hospital }\end{array}$ \\
\hline $\mathbf{9}$ & I feel able to work with people who have a diagnosis of dementia \\
\hline
\end{tabular}

Table 2. Items of the Knowledge in Dementia (KIDE) Scale

\begin{tabular}{|l|l|}
\hline $\mathbf{1}$ & Permanent changes to the brain occur in most types of dementia \\
\hline $\mathbf{2}$ & People who have dementia will usually show the same symptoms \\
\hline $\mathbf{3}$ & Dementia can be caused by a number of small strokes \\
\hline $\mathbf{4}$ & Currently, most types of dementia cannot be cured \\
\hline $\mathbf{5}$ & When people with dementia walk around it is usually aimless \\
\hline
\end{tabular}




\begin{tabular}{|c|c|}
\hline 6 & $\begin{array}{l}\text { People with dementia will eventually lose all their ability to } \\
\text { communicate }\end{array}$ \\
\hline 7 & $\begin{array}{l}\text { People with dementia who are verbally aggressive nearly always } \\
\text { become physically aggressive }\end{array}$ \\
\hline 8 & $\begin{array}{l}\text { Brain damage is the only factor that is responsible for the way } \\
\text { people with dementia behave }\end{array}$ \\
\hline 9 & It is possible to catch dementia from other people \\
\hline 10 & $\begin{array}{l}\text { My perception of reality may be different from that of a person } \\
\text { with dementia }\end{array}$ \\
\hline 11 & People with dementia never get depressed \\
\hline 12 & $\begin{array}{l}\text { Anger and hostility occur in dementia mostly because the } \\
\text { "aggression" part of the brain has been affected }\end{array}$ \\
\hline 13 & $\begin{array}{l}\text { Dementia is a general term which refers to a number of different } \\
\text { diseases }\end{array}$ \\
\hline 14 & $\begin{array}{l}\text { A person with dementia's history and background play a } \\
\text { significant part in their behaviour }\end{array}$ \\
\hline 15 & $\begin{array}{l}\text { Physical pain may result in a person with dementia becoming } \\
\text { aggressive or withdrawn }\end{array}$ \\
\hline 16 & $\begin{array}{l}\text { A person with dementia is less likely to receive pain relief than a } \\
\text { person without dementia when they are in hospital }\end{array}$ \\
\hline
\end{tabular}

Table 3. Number of staff who fell within (preliminary) categories on the CODE scale $(n=468)$

\begin{tabular}{|l|l|l|}
\hline & Pre-training & Post-training \\
\hline Not confident & $7 \%(n=31)$ & $2 \%(n=11)$ \\
\hline Somewhat confident & $82 \%(n=373)$ & $39 \%(n=185)$ \\
\hline Very confident & $11 \%(n=50)$ & $57 \%(n=268)$ \\
\hline
\end{tabular}


Figure 1. Overview of the training programme and its materials

1. "Getting to Know Me": Manual for Trainers.

2. "Getting to Know Me": A Booklet for Staff.

3. Communication skills mini-guide - a pocket guide containing 'top tips' for communication with patients with dementia.

4. "Getting to Know Me" card - a four-sided patient document to be completed by a person and their family. The card is designed to stand up by the hospital bedside.

5. Six PowerPoint slide presentations.

6. Interview clips with people with dementia and a relative.

The main topic areas covered within the programme:

- Dementia: an introduction.

- Seeing the whole person.

- Communication.

- The impact of the hospital environment.

- Knowing the person.

- A person-centred understanding of behaviour that challenges. 
Figure 2. CONSORT diagram depicting flow of participants through the study

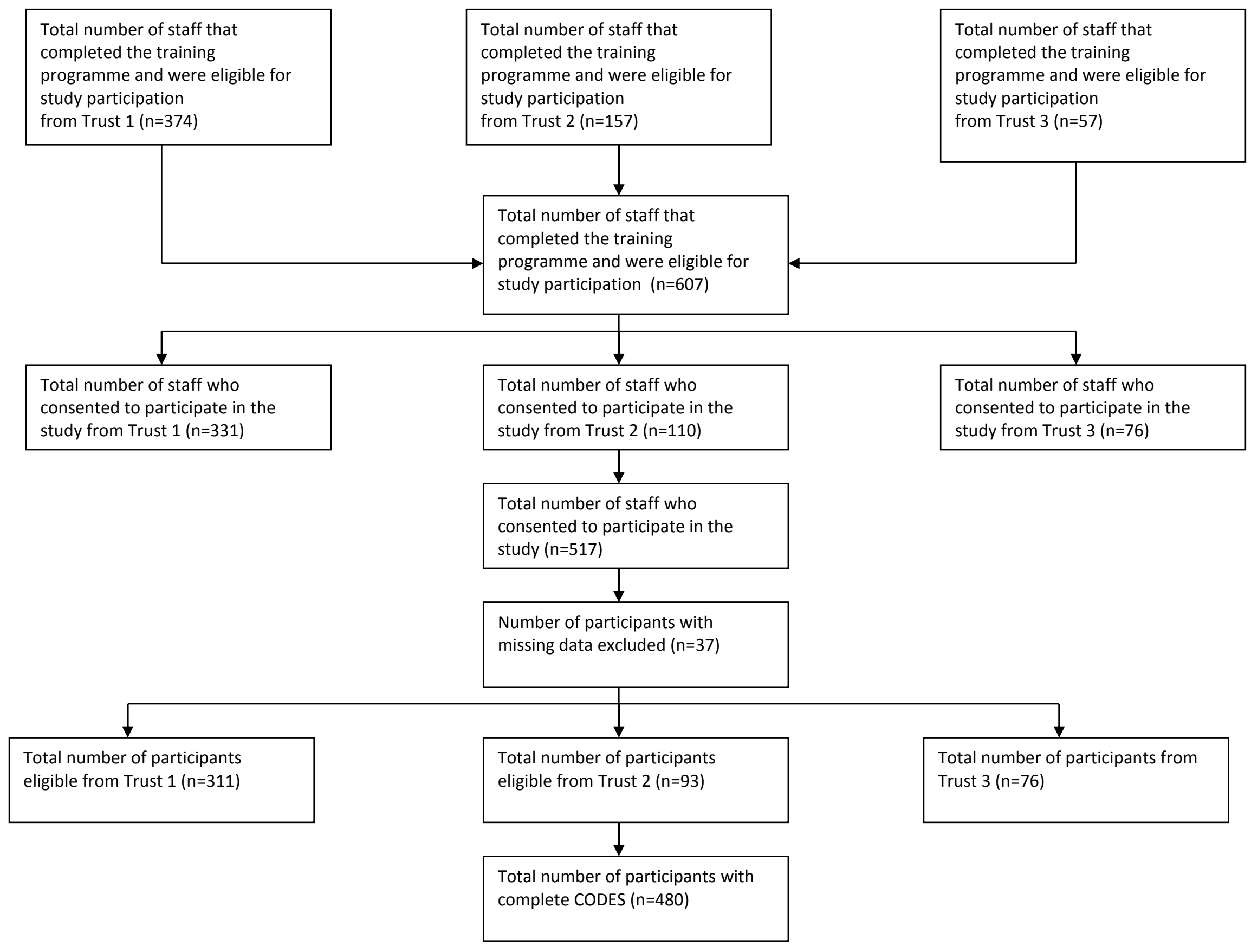


Figure 3. CONSORT diagram depicting data eligible for psychometric analysis

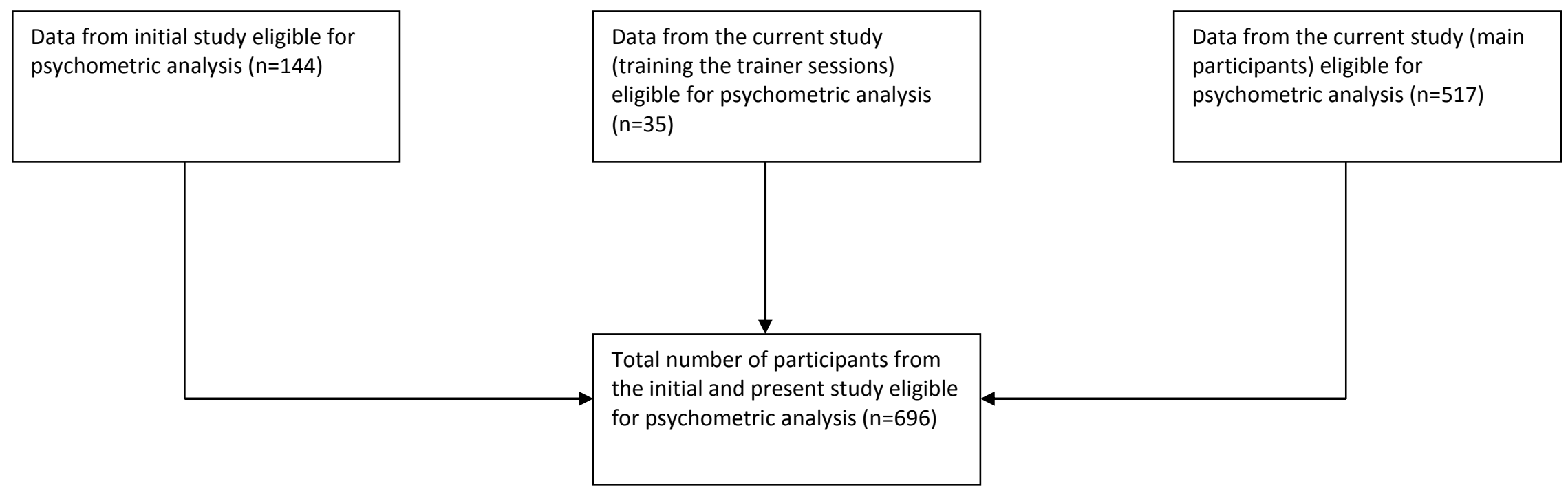


TO DO

Need to check my interpretations of effect sizes ( $r$ ). 